Piotr Kowalski

\title{
SOGNANDO LA CALIFORNIA. LE TRADUZIONI ITALIANE DELLE CANZONI BEAT
}

\author{
Sognando la California. Italian translations of beat song lyrics
}

\begin{abstract}
The article deals with song lyrics translation which was a common thing in Italy in the 1960s, the era of beat music. As a matter of fact, this new genre inspired by British and American groups such as The Beatles found fertile ground in the country which had just went through a period of prosperity which led to a rise of the middle class able to afford a growing number of luxury goods such as records. Many Italian performers preferred to copy foreign hits and remake them with Italian lyrics instead of composing new songs. The article deals with this phenomenon and proposes an analysis of three chosen song translations.

Key words: 1960s in Italy, beat music, Italian economic miracle, music translation, song lyrics

Parole chiave: gli anni '60 in Italia, miracolo economico italiano, testi delle canzoni, traduzione della musica

Słowa kluczowe: muzyka beat we Włoszech, przekład muzyczny, teksty piosenek, włoski cud gospodarczy

\section{Introduzione}

Il presente articolo ha lo scopo di avvicinare il problema delle traduzioni delle canzoni beat dall'inglese in italiano e di proporre un'analisi di alcune traduzioni prescelte, concentrandosi sulle tecniche traduttive adoperate dagli autori e sulle discrepanze più spiccanti tra il testo di partenza e quello finale. Si tratta della musica giovanile del periodo che comincia approssimativamente nel 1963 e finisce nel 1968, ispirata alle icone degli anni Sessanta come i Beatles, i Rolling Stones o cantautori come Bob Dylan. La loro stragrande popolarità e la ancora piuttosto scarsa conoscenza della lingua inglese da parte degli italiani in quell'epoca ${ }^{1}$ sono

${ }^{1}$ B. Severgnini, English is very sexy (evitarlo è impossibile). Ieri le canzoni da tradurre, oggi le fiction sottotitolate. Cosi cambia l'apprendimento, "Corriere della Sera", 8 giugno 2014, disponibile su: http://lettura.corriere.it/english-is-very-sexy/ (accesso: 21.11.2019).
\end{abstract}


le cause principali della proliferazione delle traduzioni dei testi di canzoni che in Italia assunse un livello difficilmente superato da qualsiasi altro paese. Al punto tale che alcuni successi provenienti dal mondo anglosassone furono riproposti dagli artisti italiani in diverse versioni tradotte.

\section{Una breve caratteristica del beat}

Gli anni Sessanta costituirono il palcoscenico di molte trasformazioni importanti sia in Italia che nel mondo intero. In molti paesi occidentali la dinamica della crescita economica raggiunse il livello particolarmente alto, traducendosi in un allargamento della classe media la cui condizione finanziaria permetteva di godere di più delle nuove tecnologie sotto varie forme: mezzi di trasporto, di comunicazione di massa, elettrodomestici ed altri impianti, anche a funzioni culturali come, a titolo di esempio, i giradischi e magnetofoni.

Questa situazione economica a cavallo degli anni Cinquanta e Sessanta in Italia assunse le dimensioni di un vero "boom economico" diventato mitico a causa di molti brillanti successi italiani diffusi in gran parte del mondo e diventati oggetti di culto come i famosi scooter Vespa e Lambretta, le lussuose macchine sportive Ferrari e i film di registi come Antonioni, Fellini e Visconti. Infatti, il Bel Paese in quegli anni godeva di rispetto e di interesse internazionale maggiori se paragonato con i decenni successivi.

Ma gli anni Sessanta, preceduti da un grandissimo boom demografico del secondo dopoguerra, furono anche un'epoca nella quale appare, per la prima volta in assoluto, un fenomeno culturale mai visto prima, ossia un genere musicale fatto apposta per i giovani e dai giovani: il beat, conosciuto anche in molti altri paesi sotto vari nomi. Per di più, non si tratta solamente di uno stile musicale ma di un fenomeno con tutta una cultura circostante composta da raduni, riviste, emissioni radiofoniche, film e movimenti di protesta e contestazione in gran parte responsabili per i fatti del memorabile anno 1968.

L'Italia, appena trascorso il picco del miracolo economico, si rivelò una terra molto fertile per questa nuova rivoluzione culturale. Fertile al punto tale che uno di numerosi storici e ricercatori del beat italiano, Claudio Pescetelli, elenca nella sua antologia dei gruppi beat attivi negli anni 1964-1970 pressoché cinquecento complessi e questa cifra riguarda solamente quelli dei quali si conservarono incisioni discografiche ${ }^{2}$.

Nonostante che sia un fenomeno in gran parte importato dall'oltremanica, il beat italiano è ben caratteristico sotto molti aspetti. Nessun altro paese può vantare una tale quantità delle incisioni dei successi originalmente cantati

2 C. Pescetelli, Una generazione piena di complessi. Miti e meteore del beat italiano, Zona, Civitella Val di Chiana 2006, p. 173-177. 
in inglese con i nuovi testi in lingua nazionale. Alcuni successi dei Beatles o i Rolling Stones furono persino indipendentemente riproposti in tre diverse versioni da complessi provenienti da località differenti. Inoltre i gruppi inglesi più importanti come i sopramenzionati Rolling Stones o gli Yardbirds ${ }^{3}$ incisero in italiano: i primi nel 1965 riproposero un loro successo As Tears Go By in versione italiana, intitolata Con le mie lacrime, i secondi parteciparono al famoso Festival di San Remo nel 1966 con la canzone Questa volta scritta da Gianni Marchetti, Mogol e Roberto Satti.

La proliferazione delle canzoni inglesi tradotte in italiano può sembrare strana vista l'incompatibilità della sintassi e del sistema fonetico italiani con la lingua inglese, soprattutto a causa dell'intonazione assai particolare, il grado di apertura delle vocali e l'economia sintattica.

Inoltre, l'epoca del beat costituisce un periodo problematico per i parolieri italiani a causa della divergenza tra il ritmo della lingua e quello della musica, soprattutto per la mancanza delle parole ossitone e il cambiamento della sensibilità linguistica del pubblico. Le apocopi innaturali, indispensabili nelle strutture metriche tipiche per l'italiano diventarono inaccettabili ${ }^{4}$.

Lapparente mancanza dell'originalità musicale di molti artisti beat, i quali preferivano tradurre le canzoni che avevano già avuto successo all'estero piuttosto che comporre da capo, è dovuta a un altro tratto caratteristico del fenomeno, cioè la sua forte commercializzazione. Questo fatto risulta paradossale se si prende in considerazione il versante ribelle del movimento, il quale mette in dubbio la realtà consumista e va verso l'ideologia degli hippies ${ }^{5}$. Il meccanismo della scelta delle canzoni da tradurre o localizzare fu descritto in una intervista rilasciata da Maurizio Vandelli, il vocalista chitarrista del gruppo modenese Equipe 84, uno dei maggiori esponenti del beat italiano:

Tutti noi musicisti di notte ci mettevamo ad ascoltare Radio Luxembourg o Radio Caroline che trasmetteva da una nave. In questo modo facevamo i talent scout, scoprivamo cioè i pezzi, dato che nessuno ci diceva «questo è il disco che vende

${ }^{3}$ Gli Yardbirds furono un complesso rhythm'n'blues e beat inglese che diede inizio alla carriera di tre fra i più grandi chitarristi nella storia della musica rock e blues: Eric Clapton, Jeff Beck e Jimmy Page.

${ }^{4}$ L. Zuliani, New directions in Italian song lyrics?, [in:] Text and Tune. On the Association of Music and Lyrics in Sung Verse, a cura di T. Proto, P. Canettieri, G. Valenti, Peter Lang, Bern 2015, p. 259.

5 Paradossalmente il versante più insubordinato del movimento beat è spesso identificato con le canzoni come Un ragazzo di strada incisa nel 1966 dal complesso emiliano I Corvi, nella quale viene confrontata la situazione materiale di una ragazza appartenente alla classe media borghese e quella di un semplice "ragazzo di strada". Ma la canzone è, a sua volta, pure una traduzione di un successo americano Ain't No Miracle Worker del gruppo The Brogues. 
di più in Inghilterra in questo momento». Per cui sentivamo un pezzo, ad esempio «Bang Bang», magari per trenta secondi e poi più niente, perché ascoltavamo la radio in onde lunghe, e la ricezione andava e veniva. Così certe volte cominciavamo a montare un pezzo senza sapere magari poi come finiva. Nel frattempo mandavamo qualcuno alla ricerca del disco originale e poi lo rifacevamo subito in italiano [...]. L'ascolto di Radio Luxembourg serviva quindi anche per affinare il gusto, per restare informati su cosa succedeva all'estero ${ }^{6}$.

\section{La problematica delle traduzioni di canzoni}

Le traduzioni legate al mondo musicale costituiscono un fenomeno ampiamente studiato sotto vari aspetti. Rientrano nell'area di interesse della traduttologia letteraria e quella audiovisiva, che si sono rivelate discipline molto fertili negli ultimi anni se si prende in considerazione il numero di varie pubblicazioni recenti. Ma la maggioranza di quegli studi si concentra sulla musica lirica, al massimo sulla canzone tradizionale oppure sui musical ${ }^{7} \mathrm{che}^{\mathrm{c}}$ sono forse ritenuti degli oggetti di ricerca più seri. Infatti sono piuttosto scarsi gli studi che si concentrerebbero sulla musica popolare italiana del Novecento nonostante la proliferazione delle traduzioni di canzoni in quel periodo che non interessa solamente la musica beat ${ }^{8}$, ma va dal primo dopoguerra sino alla metà degli anni Settanta.

Il presente articolo tratta delle traduzioni chiaramente classificabili come poetiche e non audiovisive siccome non si ha a che fare con la traduzione intersemiotica secondo la classificazione di Roman Jakobson ${ }^{9}$. La traduzione della poesia fu per molti anni un argomento controverso, alcuni studiosi e poeti

6 Parole di Maurizio Vandelli citate in: L. Ceri, E. De Pascale, Mondo beat. Musica e costume nell'Italia degli anni Sessanta, Fuori Thema, Bologna 1993, p. 22.

7 Un esempio molto esplicito di quello spettro di interessi assai ristretto è una raccolta di saggi Music, Text and Translation che dedica ampio spazio alla problematica di traduzioni legate all'opera lirica e generi associati. Un capitolo è dedicato ai musical con Marilyn Monroe. Le traduzioni della musica popolare sono un argomento completamente ignorato nella pubblicazione. Cfr. Music, Text and Translation, ed. H. J. Minors, Bloomsbury Academic, London 2012.

8 Il movimento beat fu attivo solo per pochi anni, in Italia si tratta approssimativamente del quinquennio dal 1963 (quando nel paese giungono le prime canzoni dei Beatles, suscitando un enorme interesse fra i giovani) al 1968 (molti complessi beat italiani cessano la loro attività, altri affrontano delle difficoltà che gli fanno cambiare direzione musicale).

9 R. Jakobson, Aspetti linguistici della traduzione, [in:] Saggi di linguistica generale, a cura di L. Heilmann, Feltrinelli, Milano 1970, p. 57. 
come Benedetto Croce parlavano persino dell'impossibilità delle traduzioni poetiche e non senza ragione.

Ogni traduzione $[. .$.$] o sminuisce e guasta, ovvero crea una nuova espressione,$ rimettendo la prima nel crogiuolo e mescolandola con le impressioni personali di colui che si chiama traduttore. Nel primo caso l'espressione resta sempre una, quella dell'originale, essendo l'altra più o meno deficiente, cioè non propriamente espressione: nell'altro saranno sì due, ma di due contenuti diversi ${ }^{10}$.

Ma le traduzioni poetiche per musica aggiungono ulteriori problemi. Il testo non deve essere soltanto fedele (che, come mostrerà l'analisi delle traduzioni del capitolo seguente, non era lo scopo principale dei traduttori delle canzoni beat) ma deve, soprattutto, conservare la metrica originale per poter funzionare bene con il ritmo e la linea melodica. Nella musica beat questi due fattori giocano il ruolo importantissimo, anzi sono più importanti del contenuto letterario. Come altrimenti spiegare il fenomeno dello stragrande primato dei gruppi inglesi affermatosi in quegli anni in gran parte del mondo nell'epoca quando ancora la conoscenza della lingua inglese da parte dei destinatari delle loro incisioni era piuttosto scarsa?

Ciononostante, sarebbe molto sbagliato constatare che i testi delle canzoni classificabili come beat non hanno nessuna importanza letteraria e servono soltanto per renderle più orecchiabili. Basta pensare al Premio Nobel per la letteratura del 2016, attribuito a Bob Dylan, "per aver creato nuove espressioni poetiche all'interno della grande tradizione della canzone americana”. L'artista americano diventò una delle figure di spicco nel movimento beat mondiale intorno alla metà degli anni Sessanta, dopo la sua mitica "elettrificazione"11.

Per di più, se non per il messaggio contenuto nelle parole delle canzoni, il beat non avrebbe mai avuto un tale impatto sulla cultura italiana nonché su quella europea, al punto tale da contribuire all'esordio della prima completa sottocultura giovanile moderna: i capelloni. Cessando così di "essere solo uno spettacolo e facendosi portavoce [...] della collettività"12. Tutti questi fattori devono essere presi in considerazione durante l'analisi delle traduzioni delle canzoni di quell'epoca.

10 B. Croce, Estetica come scienza dell'espressione e linguistica generale. Teoria e storia, a cura di G. Galasso, Adelphi, Milano 1990, p. 87.

11 Si tratta del brusco passaggio dalla musica folk con l'accompagnamento della chitarra acustica e armonica a bocca alle sonorità elettriche del rock, avvenuto durante il Newport Folk Music Festival del 1965. Cfr. E. Wald, Dylan Goes Electric! Newport, Seeger, Dylan, and the Night that Split the Sixties, Dey Street Books, New York 2015.

12 T. Tarli, Beat italiano dai capelloni a Bandiera Gialla, Castelvecchi, Roma 2007, p. 100. 


\section{L'analisi delle traduzioni}

Al fine di analisi delle traduzioni sono state scelte tre canzoni: Con le mie lacrime incisa dai Rolling Stones nel 1965, versione italiana di uno dei primi brani composti e scritti per il famoso duetto Mick Jagger e Keith Richards, As Tears Go By, interpretato per la prima volta da Marianne Faithfull; Sognando la California incisa nel 1966 dal complesso milanese I Dik Dik, versione italiana di California Dreamin' dei Mama's and the Papa's e Ti voglio, versione italiana di I Want You di Bob Dylan, incisa nel 1967 dal gruppo emiliano beat I Nomadi. Questa selezione è dovuta alla volontà di coprire i diversi versanti del movimento beat: Con le mie lacrime ne rivela il lato commerciale, essendo una canzone molto lontana dalle caratteristiche normalmente associate all'opera e alla tenuta da palcoscenico dei Rolling Stones; Sognando la California ne esprime il versante esistenzialista; Ti voglio è un esempio della poesia beat a pieno titolo.

La prima delle traduzioni sopraelencate è un'opera di Dante Panzuti, paroliere italiano, l'autore di varie traduzioni dei successi dei complessi inglesi come i Beatles. Già dai primi versi si nota l'apocope delle vocali atoni finali, tratto molto comune della tradizione poetica italiana. La sillabazione del testo tradotto rivela alcune differenze rispetto all'originale. Per esempio, il verso Dei bimbi corrono a giocar è composto da 9 sillabe e non da 8 come nell'originale. Ma l'ascolto dell'incisione chiarisce il dubbio: si tratta di una sinalefe del predicato con il suo complemento di fine. Per di più, differisce anche la costruzione metrica delle strofe. Infatti, la seconda strofa dell'originale presenta due versi di otto sillabe, due versi di sette e due di quattro, mentre tutte le strofe della traduzione hanno la costruzione identica di due ottonari, due settenari, un quadrisillabo e un ottonario finale che corrisponde a due quadrisillabi della versione inglese. Vi sono anche alcune lacune semantiche nella traduzione italiana, legate alla sinteticità dell' inglese la quale permette di esprimere più concetti utilizzando lo stesso numero di parole. Perciò il concetto della pioggia espresso nella seconda strofa All I hear is the sound / Of rain falling on the ground è completamente trascurato nella versione italiana. Ciononostante la funzione emotiva della canzone è ben preservata.

La seconda è dovuta a $\mathrm{Mogol}^{13}$, uno dei maggiori parolieri nella storia della canzone italiana moderna. Sin dall'inizio si nota un leggero ma cospicuo allontanamento semantico dall'originale, il che non cambia il senso e la funzione emotiva del testo ma ne altera i dettagli. Per esempio, i primi due versi del componimento sono in ordine inverso. I seguenti divergono

${ }^{13}$ Mogol è lo pseudonimo artistico di Giulio Rapetti, paroliere, scrittore e produttore discografico italiano, conosciuto soprattutto per la su fortunata collaborazione con Lucio Battisti. 
ulteriormente dall'originale, pur conservando l'immagine poetica della stagione particolarmente grigia e rigida la quale incita a sognare la California con il suo caldo sole. Curiosamente, il testo italiano non precisa di quale stagione si tratta e le foglie gialle invece di leaves are brown fanno pensare piuttosto all'autunno che all'inverno esplicitamente espresso in California Dreamin'. Per quanto alla struttura metrica, la traduzione cerca di seguire fedelmente il testo di partenza, ma, a causa della sinteticità dell'inglese già menzionata, alcuni versi presentano una sillaba in più (sono solitamente sei invece di cinque dell'originale) che nell'esecuzione musicale viene mascherata con ricorso a sinalefe. Un altro espediente curioso presente nella traduzione è che i versi della seconda voce non ripetono esattamente il verso che seguono, il che non avviene nell'originale; per esempio, dopo il verso Sento solo freddo la seconda voce echeggia tanto freddo sai.

Il terzo caso, quindi la canzone di Bob Dylan I Want You tradotta in italiano da un altro paroliere italiano di spicco, Giorgio Calabrese, presenta ulteriori difficoltà traduttive. Si tratta di un testo molto allusivo, pieno di metafore originali e chiaramente ispirato agli autori appartenenti alla Beat Generation americana come Allen Ginsberg. E già dalla prima lettura risulta evidente che il traduttore ha compiuto un lavoro particolare per quanto riguarda la trasposizione dell'immaginario semantico del cantautore statunitense. Ciò non sarebbe stato possibile se non per alcuni ovvi compromessi che servono a creare un prodotto comprensibile per i destinatari di lingua italiana nonché limare le differenze dovute ai diversi livelli di sinteticità rappresentati dall'inglese e italiano. Così furono eliminate alcune metonimie: i sassofoni d'argento (silver saxopohones) diventarono le orchestre e Queen of Spades diventò il solito motel. Nella traduzione di Calabrese sono numerose le modifiche strutturali, ad esempio il soggetto dell'inizio della seconda strofa the drunken politician diventò plurimo politicanti, alcolizzati; poi nella stessa strofa And I wait for them to interrupt / Me drinkin' from my broken cup venne tradotto come io aspetto chi allontanerà / l'amaro calice da me. Alcune scelte del traduttore si rivelano ancora più divergenti dal testo di partenza. In particolar modo lo si nota nell'ultima strofa. Lì, your dancing child with his Chinese suit he spoke to me diventa Il tuo bamboccio l'ho incontrato. Da notare che, nonostante gli ovvi cambiamenti, Calabrese preservò la dislocazione a sinistra del complemento. Ma il cambiamento più drastico si trova verso la fine della strofa e della canzone. Invece di tradurre il testo originale fino in fondo, il traduttore scelse di sostituire il contenuto a partire della metà dell'ultima strofa ripetendo gli ultimi tre versi della prima. Al livello metrico, l'analisi non rivela delle caratteristiche sorprendenti ma qualche volta i versi italiani risultano leggermente più lunghi per quanto riguarda il numero di sillabe. Ma anche la canzone originale è, come molti altri componimenti del recente laureato del Premio Nobel, irregolare quanto alla metrica. Basta guardare allo schema delle rime il quale è spezzato, sia nella versione originale che nella traduzione di 
Calabrese. Per di più, lingua inglese possiede un maggior numero delle parole monosillabe. Infatti, secondo Werner Gitt, la percentuale di tali parole in inglese è di $71,5 \%{ }^{14}$. Ciò impone la necessità della riduzione semantica nelle traduzioni poetiche.

Tabella 1

\begin{tabular}{|l|l|}
\hline $\begin{array}{l}\text { Con le mie lacrime } \\
\text { Musica: Mick Jagger e Keith Richards }\end{array}$ & $\begin{array}{l}\text { As Tears Go By } \\
\text { Muducica e testo: Mick Jagger e Keith } \\
\text { Richards }\end{array}$ \\
Il sole sta per tramontar & It is the evening of the day \\
Visi che sorridono & I sit and watch the children play \\
Ed io son qui & Smiling faces I can see \\
Con le mie lacrime cosi & But not for me \\
& I sit and watch \\
Con la ricchezza io potrei & As tears go by \\
Comprare quello che vorrei & My riches can't buy everything \\
Ma la gioia semplice & I want to hear the children sing \\
Perciò son qui & All I hear is the sound \\
Con le mie lacrime cosi & Of rain falling on the ground \\
& I sit and watch \\
Il sole sta per tramontar & As tears go by \\
Un altro giorno se ne va & It is the evening of the day \\
Tutti si divertono & I sit and watch the children play \\
Ed io son qui & Doing things I used to do \\
Con le mie lacrime cosi & They think are new \\
& I sit and watch \\
As tears go by \\
\hline
\end{tabular}

14 W. Gitt, In the Beginning Was Information. Scientist Explains the Incredible Design in Nature, Master Books, Green Forrest 2007, p. 201. 
Tabella 2

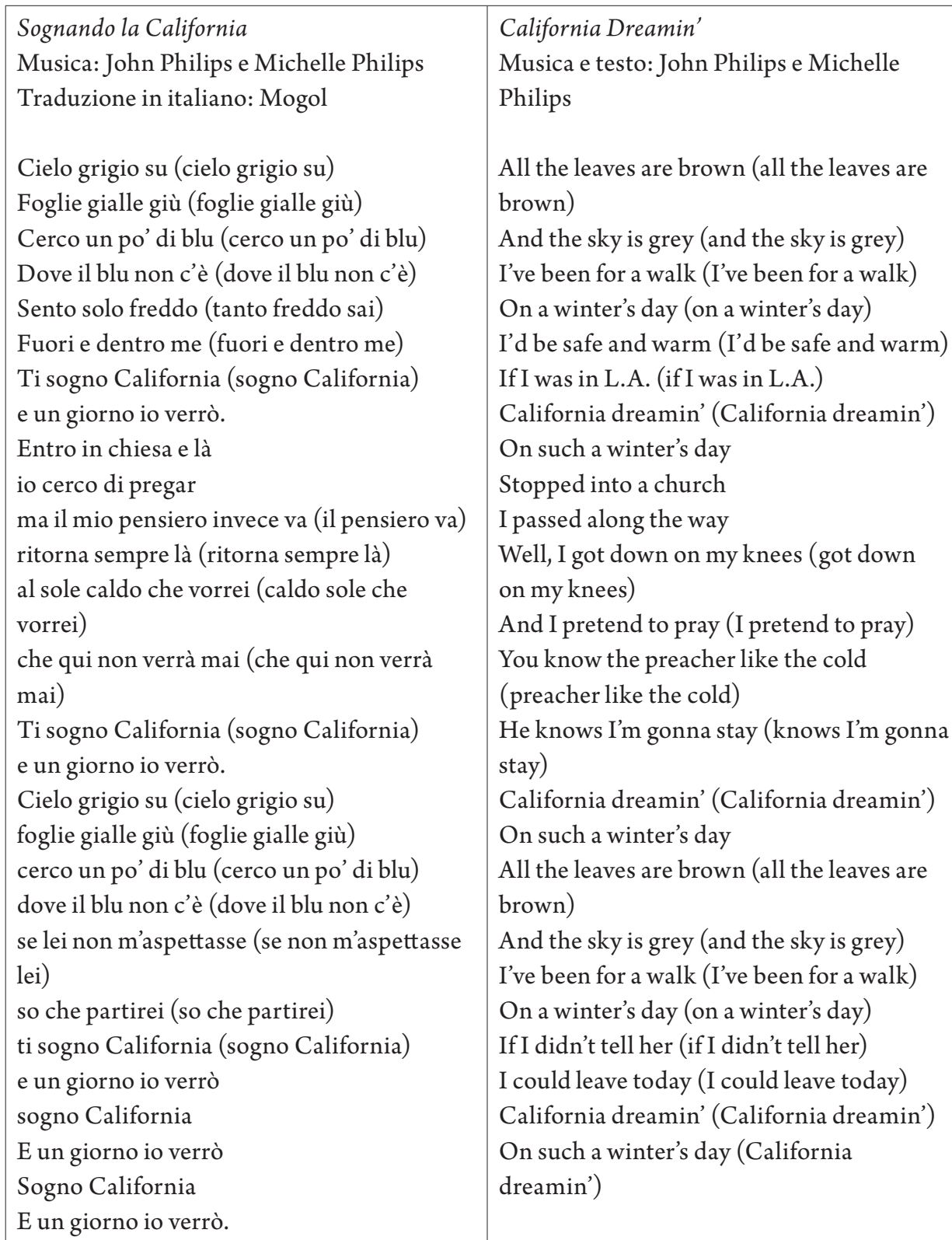


Tabella 3

\begin{tabular}{|c|c|}
\hline $\begin{array}{l}\text { Tivoglio } \\
\text { Musica: Bob Dylan } \\
\text { Traduzione in italiano: Giorgio Calabrese }\end{array}$ & $\begin{array}{l}\text { I Want You } \\
\text { Musica e testo: Bob Dylan }\end{array}$ \\
\hline $\begin{array}{l}\text { Falsi sospiri di becchini, } \\
\text { di suonatori di organino } \\
\text { le orchestre dicon che dovrei lasciarti, } \\
\text { campane in festa e trombe d'auto } \\
\text { sghignazzano in faccia a me } \\
\text { è ingiusto sai se ti perdo. }\end{array}$ & $\begin{array}{l}\text { The guilty undertaker sighs } \\
\text { The lonesome organ grinder cries } \\
\text { The silver saxophones say I should refuse } \\
\text { you } \\
\text { The cracked bells and washed-out horns } \\
\text { Blow into my face with scorn, but it's } \\
\text { Not that way, I wasn't born to lose you }\end{array}$ \\
\hline $\begin{array}{l}\text { Ti voglio, ti voglio, ti voglio con me } \\
\text { quanto ti voglio }\end{array}$ & $\begin{array}{l}\text { I want you, I want you, I want you, so bad } \\
\text { Honey, I want you }\end{array}$ \\
\hline $\begin{array}{l}\text { Politicanti, alcolizzati } \\
\text { schivano gli occhi di affamati } \\
\text { e un redentore da due soldi, ti aspetta } \\
\text { io aspetto chi allontanerà } \\
\text { l'amaro calice da me } \\
\text { e mi dirà di aprir la porta a te. }\end{array}$ & $\begin{array}{l}\text { The drunken politician leaps } \\
\text { Upon the street where mothers weep } \\
\text { And the saviors who are fast asleep, they } \\
\text { wait for you } \\
\text { And I wait for them to interrupt } \\
\text { Me drinkin' from my broken cup } \\
\text { And ask me to open up the gate for you }\end{array}$ \\
\hline $\begin{array}{l}\text { Ti voglio, ti voglio, ti voglio con me. } \\
\text { Quanto ti voglio? }\end{array}$ & $\begin{array}{l}\text { I want you, I want you, yes I want you, so } \\
\text { bad } \\
\text { Honey, I want you }\end{array}$ \\
\hline $\begin{array}{l}\text { I padri non ci sono più } \\
\text { il vero amore è sotterrato } \\
\text { e i figli han disdegnato me } \\
\text { perché non vi ho pensato. } \\
\text { Ritorno al solito motel } \\
\text { la cameriera è sempre là } \\
\text { ed è gentile come sempre, con me } \\
\text { lei vede tutto, e sa già } \\
\text { che io vorrei essere con te } \\
\text { ma non dice niente, lei sa che } \\
\text { Ti voglio, ti voglio, ti voglio con me. } \\
\text { Quanto ti voglio. }\end{array}$ & $\begin{array}{l}\text { How all my fathers, they've gone down } \\
\text { True love they've been without it } \\
\text { But all their daughters put me down } \\
\text { 'Cause I don't think about it } \\
\text { Well, I return to the Queen of Spades } \\
\text { And talk with my chambermaid } \\
\text { She knows that I'm not afraid to look at her } \\
\text { She is good to me and there's } \\
\text { Nothing she doesn't see } \\
\text { She knows where I'd like to be but it doesn't } \\
\text { Matter } \\
\text { And because time was on his side and } \\
\text { Because I }\end{array}$ \\
\hline
\end{tabular}




\begin{tabular}{|l|l|}
\hline $\begin{array}{l}\text { Il tuo bamboccio l'ho incontrato } \\
\text { ancora un po' e l'avrei strozzato } \\
\text { non son stato furbo, lo sai }\end{array}$ & $\begin{array}{l}\text { I want you, I want you, yes I want you, so } \\
\text { bad. }\end{array}$ \\
campane in festa e trombe d'auto & Honey, I want you \\
sghignazzano in faccia a me & Now your dancing child with his Chinese \\
è ingiusto sai, se ti perdo. & suit he \\
& Spoke to me, I took his flute \\
Ti voglio, ti voglio, ti voglio con me. & No, I wasn't very cute to him, was I? \\
Quanto ti voglio? & But I did it, because he lied and \\
& Because he took you for a ride \\
& want you, I want you, Yes I want you, so bad. \\
& Honey, I want you \\
\hline
\end{tabular}

\section{Conclusioni}

Nonostante che il beat sia un fenomeno che è durato pochi anni, il suo peso storico è irrefutabile. Una gran parte del suo impatto sulla storia della società occidentale sta nel fatto che fu il primo genere musicale a provocare un interesse cosi enorme tra i giovani. I giovani che per la prima volta si rivelarono una generazione molto diversa non solamente dagli altri strati della popolazione, ma diversa anche dai giovani di prima.

Riassumendo quanto detto prima, la musica beat differisce in modo considerevole sia dai suoi punti di riferimento anglosassoni che dalle sue diverse varietà europee, assumendo un chiaro carattere nazionale dal punto di vista della scelta delle canzoni, produzione musicale, lingua e tematica dei testi contribuendo all'identità culturale dell'Italia moderna.

Ciononostante, le influenze inglesi e americani fanno sì che con la musica beat si rompa definitivamente con il primato della lingua italiana come la lingua della musica ascoltata in Italia. Per la prima volta nella storia, il pubblico italiano, soprattutto quello giovanile, si apre così tanto alle canzoni in lingua inglese. Inoltre in quel epoca finisce il già indebolito primato internazionale dell'italiano nel campo musicale che si cristallizzò nel Settecento con il successo dell'opera di Pietro Metastasio, in cui opere si vedono chiaramente due tratti che fecero dell'italiano la lingua più adatta alla produzione musicale: il ritmo facile delle arie e la sintassi semplice ed elementare, subordinata alla musica ${ }^{15}$.

Nell'epoca del beat i successi provenienti d'oltremanica godevano di una popolarità enorme benché molti dei loro destinatari non sapessero di che cosa trattavano le parole. Ciò creò una nuova tendenza che diventò uno dei più notevoli tratti caratteristici del beat italiano che è la proliferazione delle

15 S. Morgana, Profilo di storia linguistica italiana, [in:] I. Bonomi et al., Elementi di linguistica italiana, Carocci Editore, Roma 2016, p. 235. 
traduzioni di canzoni, un fenomeno assai particolare. Come dimostra l'analisi del campione scelto, i loro autori non sempre ebbero un compito semplice, alcuni affrontarono dei testi che non erano "solo canzonette". Quelle traduzioni senz'altro influirono molto sull'immaginario collettivo di chi le ascoltava, in parte contribuendo alle convinzioni di tutta una generazione. Generazione che fece la storia partecipando attivamente negli avvenimenti del Sessantotto. Perciò non sarebbe completamente infondata la conclusione che in quel caso, la storia la fecero i traduttori, almeno in parte.

\section{Bibliografia}

Ceri L., De Pascale E., Mondo beat. Musica e costume nell'Italia degli anni Sessanta, Fuori Thema, Bologna 1993.

Croce B., Estetica come scienza dell'espressione e linguistica generale. Teoria e storia, a cura di G. Galasso, Adelphi, Milano 1990.

Gitt W., In the Beginning Was Information. Scientist Explains the Incredible Design in Nature, Green Forrest 2007.

Jakobson R., Aspetti linguistici della traduzione, [in:] Saggi di linguistica generale, a cura di L. Heilmann, Feltrinelli, Milano 1970, p. 56-64.

Morgana S., Profilo di storia linguistica italiana, [in:] I. Bonomi et al., Elementi di linguistica italiana, Carocci Editore, Roma 2016, p. 189-299.

Music, Text and Translation, ed. H. J. Minors, Bloomsbury Academic, London 2012.

Pescetelli C., Una generazione piena di complessi. Miti e meteore del beat italiano, Zona, Civitella Val di Chiana 2006.

Severgnini B., English is very sexy (evitarlo è impossibile). Ieri le canzoni da tradurre, oggi le fiction sottotitolate. Cosi cambia l'apprendimento, "Corriere della Sera", 8 giugno 2014, disponibile su: http://lettura.corriere.it/english-is-very-sexy/ (accesso: 21.11.2019).

Tarli T., Beat italiano dai capelloni a Bandiera Gialla, Castelvecchi, Roma 2007.

Wald E., Dylan Goes Electric! Newport, Seeger, Dylan, and the Night that Split the Sixties, Dey Street Books, New York 2015.

Zuliani L., New directions in Italian song lyrics?, [in:] Text and Tune. On the Association of Music and Lyrics in Sung Verse, a cura di T. Proto, P. Canettieri, G. Valenti, Peter Lang, Bern 2015, p. 255-272. 